Corrigendum

\title{
Corrections to "Optimal order of servers in a tandem queue with general blocking"
}

\author{
D.W. Cheng \\ Department of Statistics and Operations Research, 704 Tisch Hall, \\ New York University, NY 10003, USA
}

Y. Zhu

Department of Industrial Engineering, 405 Bell Hall, SUNY at Buffalo, NY 14260, $U S A$

Received 14 April 1994

In our recent paper "Optimal order of servers in a tandem queue with general blocking" published in Queueing Systems 14 (1993) 427-437, some typos and errors are found. We would like to correct them as follows:

(1) In equation (8) on page $430, C_{n+1}$ should be replaced by $C_{n}$.

(2) In lemma 3 on page $431, \cap_{j=1,3,4,5}$ should be replaced by $\cup_{j=1,3,4,5}$.

(3) Theorem 2 on page 432 should be stated as: For two systems with $a_{m-1}=k_{m-1}$ and $b_{m-1}=a_{m}=1$, if $X \leq_{r h}\left(\right.$ resp. $\left.\leq_{s t}\right) Y$ then $T H_{B_{A}} \leq T H_{B_{B}}$.

(4) $D_{n}^{i}(x, y)$ in lemma 6 should be replaced by $I_{n}^{i}(x, y)$ on page 434 .

(5) Lemma 3 should be theorem 3 on page 435. Moreover, in the lemma, $D_{n, A}^{i} \leq_{s t} D_{n, B}^{i}$ and $D_{n, A}^{i} \leq_{i d c x} D_{n, B}^{i}$ should be replaced by $I_{n, A}^{i} \leq_{s t} I_{n, B}^{i}$ and $I_{n, A}^{i} \leq_{i d c x} I_{n, B}^{i}$, respectively.

\section{Acknowledgement}

We would like to thank R. Righter who pointed out some of the errors in the paper. 